\title{
Hyperacute Detection of Neurofilament Heavy Chain in Serum Following Stroke: A Transient Sign
}

\author{
Johann Sellner • Amit Patel • Pooja Dassan • \\ Martin M. Brown • Axel Petzold
}

Received: 10 June 2011/Revised: 9 July 2011 / Accepted: 14 July 2011 / Published online: 27 July 2011

(C) Springer Science+Business Media, LLC 2011

\begin{abstract}
Serological biomarkers which enable quick and reliable diagnosis or measurement of the extent of irreversible brain injury early in the course of stroke are eagerly awaited. Neurofilaments (Nf) are a group of proteins integrated into the scaffolding of the neuronal and axonal cytoskeleton and an established biomarker of neuroaxonal damage. The $\mathrm{Nf}$ heavy chain $\left(\mathrm{NfH}^{\mathrm{SMI} 35}\right)$ was assessed together with brain-specific astroglial proteins GFAP and S100B in hyperacute stroke (6 and $24 \mathrm{~h}$ from symptom onset) and daily for up to 6 days. Twenty-two patients with suspected stroke (median NIHSS 8) were
\end{abstract}

Johann Sellner and Amit Patel contributed equally.

J. Sellner $(\square)$

Department of Neurology, Klinikum rechts der Isar, Technische

Universität München, Ismaninger Str. 22, 81675 Munich,

Germany

e-mail: sellner@1rz.tum.de

J. Sellner

Department of Neurology, Christian-Doppler-Klinik, Paracelsus Medizinische Universität, Ignaz-Harrer-Str. 79, 5020 Salzburg, Austria

A. Patel · A. Petzold

Department of Neuroinflammation, The National Hospital for Neurology and Neurosurgery, Queen Square,

London WC1N 3BG, UK

P. Dassan · M. M. Brown

Department of Brain Repair and Rehabilitation, UCL Institute of Neurology, Queen Square, London WC1N 3BG, UK

\section{A. Petzold}

Department of Neurology, VU Medical Centre,

1081 HV Amsterdam, The Netherlands recruited in a prospective observational study. Evidence for an ischaemic or haemorrhagic lesion on neuroimaging was found in 18 (ischaemia $n=16$, intracerebral haemorrhage $n=2)$. Serum $\mathrm{NfH}^{\mathrm{SMI} 35}$ levels became detectable within 24 h post-stroke $(P<0.0001)$ and elevated levels persisted over the study course. While GFAP was not detectable during the entire course, S100B levels peaked at the end of the observation period. The data indicate that significant in vivo information on the pathophysiology of stroke may be obtained by the determination of $\mathrm{NfH}^{\mathrm{SMI} 35}$. Further studies are required to evaluate whether $\mathrm{NfH}^{\mathrm{SMI} 35}$ in hyperacute stroke reflects the extent of focal ischaemic injury seen on neuroimaging or is a consequence of more diffuse neuroaxonal damage.

Keywords Neurofilament · Biomarker .

Ischaemic stroke $\cdot$ Blood-brain barrier · Pathophysiology

\section{Introduction}

Stroke is a serious condition resulting in significant mortality and long-term morbidity. Ischaemia is associated with reduction in oxygen and glucose supply within the central nervous system (CNS). When blood flow falls below a critical threshold, a cascade of biochemical reactions generating excitotoxicity, oxidative stress, inflammation, and cell death is triggered, leading to tissue infarction [1]. This detrimental process is associated with release of different cell-type specific proteins including calcium-binding protein S100B, glial fibrillary astrocytic protein (GFAP) and neurofilaments (Nf) into extracellular fluids (ECF). From the ECF, these proteins diffuse to the CSF and peripheral blood, where they can be quantified 
[2]. Increased attention has been directed to establishing the measurement of these molecules as a tool to assist in diagnosis, selection of treatments, assessment of the efficacy of treatments, and prediction of outcome of stroke [3].

In brief, GFAP is a brain-specific astrocytic intermediate filament protein, which is released upon cellular disintegration and degradation of the cytoskeleton. The determination of GFAP in the time window of 2 and $6 \mathrm{~h}$ after stroke may be helpful in the distinction of ischaemic from haemorrhagic stroke. Dvorak et al. reported that patients with intracerebral haemorrhage had significantly elevated GFAP levels in this period, whereas GFAP levels remain below the detection limit in ischaemic stroke [4]. S100B is mainly found in astrocytes and other sources include oligodendrocytes, microglia and neurons. Concentrations in cerebrospinal fluid (CSF) were found to be up to 60 times higher than in serum [5]. Importantly, previous reports indicated that S100B serum values correlate with final infarct volume when determined beyond $24 \mathrm{~h}$ after symptom onset [6-8]. In contrast to the multiple sources of S100B, neurofilaments, a group of proteins integrated into the scaffolding of the neuronal and axonal cytoskeleton, are specific to and abundant in the neuro-axonal compartment [9]. Both neurofilament light (NfL, $68 \mathrm{kDa}$ ) and heavy chains (NfH, 190-210 kDa) can be measured in CSF [10]. So far, quantification from serum is possible for $\mathrm{NfH}$ only. Significant positive associations were found for blood $\mathrm{NfH}$ levels and clinical severity, volume of the ischaemic territory and outcome when assessed 3 weeks after symptom onset [11]. There is no linear correlation for CSF and serum concentration. Assessment of S100B and NfH concentrations in ischaemic stroke revealed that CSF but not serum concentrations correlated positively with outcome on the modified Rankin scale (mRS) [5]. But samples were taken relatively late in this study (median 3 days, range $1-15$ days).

An important question for future treatment approaches is the time course of irreversible neuronal damage and the potential to quantify the extent. Not all incidents of oxygen and glucose deprivation will lead to irreversible brain injury [12]. Indeed, ischaemic lesions visualized by $\mathrm{CT}$ and even multimodal magnetic resonance imaging (MRI) in the acute phase $(<24 \mathrm{~h}$ of symptom onset) do not necessarily reflect the final infarct volume [13]. Moreover, milder ischaemic events will only lead to temporary neuronal dysfunction and may be even associated with preconditioning and increase resistance towards irreversible damage [14]. Hence, a biomarker identifying the extent of irreversible neuro-axonal injury in the critical early phase is of utmost importance. Here, we aimed at evaluating the earliest consequences of stroke by studying sequential serum samples with a panel of three biomarkers within $24 \mathrm{~h}$ of symptom onset and the subsequent period of 6 days.

\section{Materials and Methods}

\section{Patients and Sample Collection}

The study was approved by the local ethics committee. Written informed consent was obtained from the patients and when this was not possible from the next family member.

Patients admitted to the Accident and Emergency Department of University College London Hospital (UCLH) with a suspected acute cerebrovascular event with onset of symptoms $<24 \mathrm{~h}$ prior to assessment were evaluated prospectively over an 8 month period. Twenty-two patients were recruited and peripheral-venous blood was obtained upon arrival and daily between 9:00 and 10:00 a.m. over a 6-days period. The tube was left standing at room temperature for $30 \mathrm{~min}$ to allow blood clotting. The sample was then centrifuged at $1,500 \mathrm{~g}$ for $10 \mathrm{~min}$, and serum was removed and coded aliquots were stored at $80^{\circ} \mathrm{C}$. The clinical assessment consisted of the NIH stroke scale (NIHSS), the Glasgow coma scale and basic vital signs including blood pressure, temperature and peripheral oxygen saturation. Further parameters monitored were haematocrit and blood pressure. All patients were examined with a brain CT scan. Twenty patients presenting to the same Accident and Emergency Department without evidence for neurological disease were recruited as controls.

\section{Quantification of NfH, S100B and GFAP Levels}

Serum $\mathrm{NfH}^{\mathrm{SMI} 35}$ levels were measured using an enzymelinked immunoassay, as described previously $[10,15]$. The mouse monoclonal antibody SMI35 (IgG) was originally purchased from Sternberger Monoclonals Inc., and is now available through Covance Research Products (Berkeley, CA, USA). The secondary and tertiary antibodies used were the rabbit polyclonal anti-NfH IgG (Sigma, St. Lous, $\mathrm{MO} ; \mathrm{N}$ 4142) and the horseradish peroxidase (HRP)labelled swine polyclonal anti-rabbit IgG (DAKO, Copenhagen, Denmark). Adhering to the nomenclature previously set up, we indicate the captured antibodies used for $\mathrm{NfH}$ quantification in superscript $\left(\mathrm{NfH}^{\mathrm{SMI35}}\right.$ for SMI35). Serum S100B and GFAP ${ }^{\text {SMI26 }}$ levels were determined with an ELISA as described before $[16,17]$. The detection limit for the $\mathrm{NfH}^{\mathrm{SMI} 35}$ assay is $0.01 \mathrm{ng} / \mathrm{mL}$. The detection limits for GFAP ${ }^{\mathrm{SMI} 26}$ is $5 \mathrm{pg} / \mathrm{mL}$ and for $\mathrm{S} 100 \mathrm{~B}$ 
$7 \mathrm{pg} / \mathrm{mL}$. The analyst was blinded to all clinical information and measurements were done in duplicates.

\section{Statistical Analysis}

Statistical analysis was carried out using Graph Pad Prism Software Version 5.0 (San Diego, CA, USA). The nonparametric Mann-Whitney test was used for comparison of controls and stroke at baseline $(<24 \mathrm{~h})$. The KruskalWallis test with Dunn's multiple post-hoc comparison was used to compare dynamics of $\mathrm{NfH}^{\mathrm{SMI} 135}, \mathrm{~S} 100 \mathrm{~B}$ and GFAP $^{\text {SMI26 }}$ serum levels in the first 6 days after stroke onset. Two sided tests were used and a $P$ value $<0.05$ was accepted as significant.

\section{Results}

Stroke Patients and Controls

Radiological evidence for stroke was found in 18 patients. Ischaemic stroke was diagnosed in the majority $(n=16)$, whereas haemorrhagic stroke was the cause of neurological

Table 1 Baseline characteristics of the control and stroke cohort

\begin{tabular}{lllll}
\hline Characteristic & Control & Stroke & \multicolumn{2}{l}{ Stroke subtype } \\
\cline { 3 - 5 } & & & Ischaemic & Haemorrhagic \\
\hline Age, year (mean) & 40.2 & 65.5 & 69.5 & 59.5 \\
Sex (F:M) & $10: 10$ & $10: 8$ & $10: 6$ & $0: 2$ \\
NIHSS $^{\mathrm{a}}$ & - & 8 & 8 & - \\
GCS $^{\mathrm{a}}$ & 15 & 15 & 14 & 15 \\
No. & 20 & 18 & 16 & 2 \\
\hline
\end{tabular}

$S D$ standard deviation

a On admission symptoms in two. In the remaining four subjects no vascular or structural pathology was seen on MRI and lead to exclusion from further analysis. None of the patients with ischemic stroke was treated with intravenous alteplase. The baseline characteristics of the 18 stroke patients and controls are shown in Table 1.

Serum Levels of $\mathrm{NfH}^{\mathrm{SMI} 35}, \mathrm{~S} 100 \mathrm{~B}$ and GFAP ${ }^{\mathrm{SMI} 26}$ in Acute Stroke and Controls

Hyperacute stroke was defined as onset of stroke symptoms $<24 \mathrm{~h}$ before assessment and samples from 15 patients were available within this time period. $\mathrm{NfH}^{\mathrm{SMI} 35}$ levels were significantly higher in hyperacute stroke (median $0.13 \mathrm{ng} / \mathrm{mL}$, range $0-0.17$ ) compared to the control group (median $0 \mathrm{ng} / \mathrm{mL}$, range $0-0.05 ; P<0.0001$ ) (Fig. 1). Median S100B levels in hyperacute stroke were $(0.03 \mathrm{ng} / \mathrm{mL}$, range $0-0.33)$ were lower than in controls (0.09 ng/mL, range 0.05-0.32). The S100B levels in stroke patients were not statistically different from controls. GFAP $^{\text {SMI26 }}$ serum levels were below the detection limit in all the samples. Neither $\mathrm{NfH}^{\mathrm{SMI} 35}$ nor S100B levels correlated with age or NIHSS scores.

Time Course of $\mathrm{NfH}^{\mathrm{SMI} 35}$ and S100B in Acute Stroke

The time course of the three biomarkers levels over 6 days following stroke onset is shown in Table 2. Patients with confirmed stroke on neuroimaging had increased $\mathrm{NfH}^{\mathrm{SMI} 35}$ levels already within $6 \mathrm{~h}$ of symptom onset. The increase persisted over the 6 days course and peaked at day 4 but changes did not reach statistical significance compared to other time points. The increase of S100B, however, was delayed and started 3 days and peaked at 6 days post stroke. Differences were not statistically significant. GFAP remained below the detection limit in all stroke patients over the entire time course.
Fig. 1 Serum $\mathrm{NfH}^{\mathrm{SMI} 35}$ and S100B in hyperacute stroke $(<24 \mathrm{~h}$ from onset of symptoms). Box-whisker plots indicate minimum, median, max and the 25th and 75th percentiles. The horizontal bar in the dot-plot diagrams depicts the mean. n.s. not significant
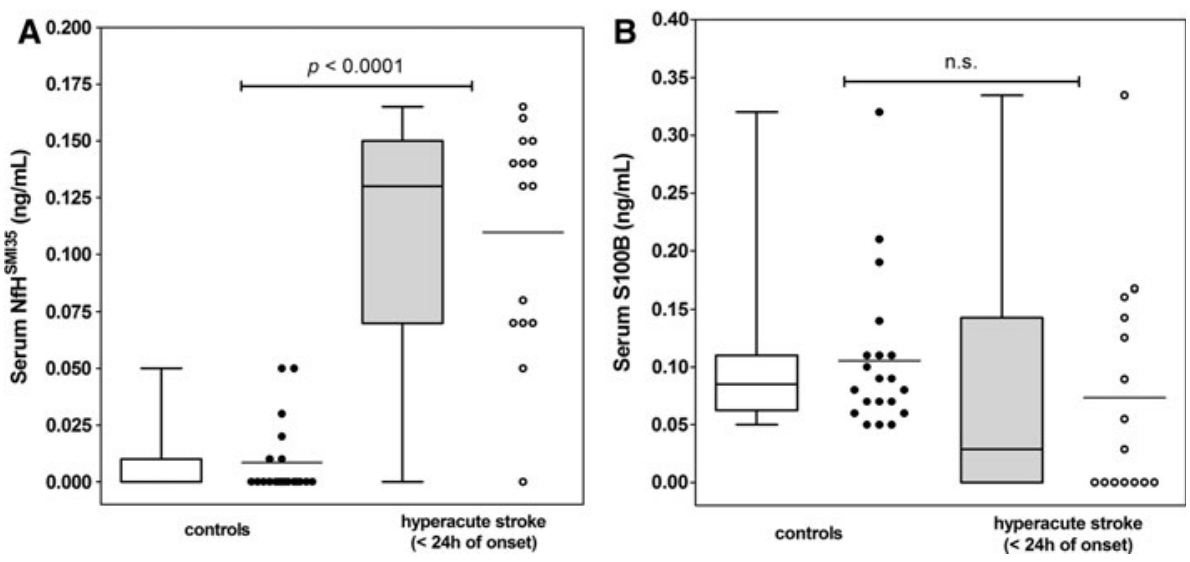
Table 2 The longitudinal profile of serum $\mathrm{NfH}^{\mathrm{SMI} 35}(\mathrm{ng} / \mathrm{mL})$ and $\mathrm{S} 100 \mathrm{~B}(\mathrm{ng} / \mathrm{mL})$ levels in stroke patients

\begin{tabular}{llllllll}
\hline \multicolumn{7}{l}{ Time (hours) after stroke } \\
\cline { 2 - 7 } & $<6 \mathrm{~h}$ & $6-24 \mathrm{~h}$ & 2 days & 3 days & 4 days & 5 days & 6 days \\
\hline No. & 8 & 7 & 10 & 7 & 8 & 11 & 10 \\
NfH $^{\text {SMI35 }}$ & $0.14(0-0.14)$ & $0.14(0-0.07)$ & $0.13(0-0.13)$ & $0.09(0-0.09)$ & $0.14(0-0.14)$ & $0.13(0-0.14)$ & $0.13(0-0.13)$ \\
S100B & $0.01(0-0.17)$ & $0.02(0-0.34)$ & $0.01(0-0.33)$ & $0.03(0-0.38)$ & $0.03(0-0.6)$ & $0.04(0-0.59)$ & $0.05(0-0.15)$ \\
GFAP & - & - & - & - & - & - & - \\
\hline
\end{tabular}

$\mathrm{NfH}^{\text {SMI35 }}$ becomes detectable already after $6 \mathrm{~h}$ from symptom onset. GFAP was below the detection limit at all time points. Median and range (brackets) are shown. Statistical analysis did not reveal significant changes over time

\section{Discussion}

This study found an extracranial increase in venous blood of $\mathrm{NfH}^{\mathrm{SMI} 35}$, a specific marker for axonal injury, degeneration and neuronal loss, already present in the very early course of acute stroke. Serum levels of $\mathrm{NfH}^{\mathrm{SMI} 35}$ became detectable within $24 \mathrm{~h}$ of symptom onset, an observation which was also verified within in the $6 \mathrm{~h}$ post-stroke time frame. These findings corroborate recent data in an experimental piglet model of global brain ischaemia due to circulatory arrest. The peak of $\mathrm{NfH}^{\mathrm{SMI} 35}$ serum level was already detected within $30 \mathrm{~min}$ after a $60 \mathrm{~min}$ course of global hypoxia [14]. The findings also extend the observation of reduced and disrupted $\mathrm{NfH}$ staining $\left(\mathrm{NfH}^{\mathrm{SMI} 1}\right.$ and silver staining) in the ischaemic hemisphere in experimental focal ischaemia starting at 1-3 days post insult [18]. Indeed, $\mathrm{NfH}^{\mathrm{SMI} 35}$ was shown to be a sensitive marker and even subtle brain injury due to symptomatic carotid stenosis was associated with 2.4-fold higher $\mathrm{NfH}^{\mathrm{SMI} 35}$ serum levels compared to asymptomatic carotid stenosis [15]. Hence, it is tempting to speculate that increased serum levels of $\mathrm{NfH}^{\mathrm{SMI} 35}$ reflect the respective acute neuro-axonal damage evidenced on neuroimaging. Such information would also be important for targeted management of stroke patients in future treatment trials. Hence, a correlation of early $\mathrm{NfH}^{\mathrm{SMI} 35}$ levels with a sensitive neuroimaging measure would be one of the next steps.

Final infarct size is determined by a combination of the initial ischaemic injury and delayed responses to the ischaemia. The latter include a number of mechanisms such as inflammatory reactions and apoptosis, which contribute to the overall brain injury [19]. This process evolves over several days and even weeks post-stroke. In keeping with this concept, Foerch and coworkers reported that serum 100B levels assessed within the early stages of stroke onset did not reflect infarct size [20]. This finding is also supported by several reports that S100B levels only correlate with final infarct volume if determined $>24 \mathrm{~h}$ after symptom onset $[6,7,21]$. Thus, Foerch et al. argued that early S100B levels may be regarded more as qualitative measure of blood-brain barrier (BBB) leakage. We are hesitant to subscribe the findings to a leakage of the BBB since this is not a precondition of increased blood concentrations of biomarkers for brain damage [22]. We previously reported a median $\mathrm{NfH}^{\mathrm{SMI} 35}$ concentration of $0.21 \mathrm{ng} / \mathrm{mL}$ in CSF of a biologically representative population recruited from the UCLH Emergency and Accident Department. This exceeds the amount found in serum by far, where $\mathrm{NfH}^{\mathrm{SMI} I 5}$ levels were below the detection limit in a group of healthy volunteers [23]. Further knowledge on release, kinetics and clearance of $\mathrm{NfH}$ across the body fluid compartments is required.

Singh et al. did not find significantly increased $\mathrm{NfH}$ values on day 1 compared to controls [11], but did not report hourly timing from stroke onset and their samples may have been taken later than in the present study. Additionally, methodological differences may apply as the analytical sensitivity in not known. Finally, our study comprised more severely affected patients with a median NIHSS of 8 compared to the 6 in the Singh et al. study. Therefore our patients are likely to have more substantial brain damage as the source for the serum $\mathrm{NfH}$ levels we measured. These issues may have accounted for the detectability of $\mathrm{NfH}^{\mathrm{SMI} 35}$ in the hyperacute phase of stroke. Compared to healthy controls, Singh reported a 2.9- and 8.5 -fold increase after 1 and 3 weeks, respectively. The further increase at the 3 weeks time point is particularly intriguing since the observation may correspond to additional neuro-axonal injury induced by post-stroke immune and inflammatory processes. We confirm previous studies that S100B is detectable very early in the course of stroke and peaks within 1 week in nonfibrinolytic-treated stroke patients $[7,24,25]$. Yet, S100B in our study did not differ from the control group, which could be traced back to the small sample size. In contrast, GFAP could not be detected over the entire study period. In this regard, Herrmann and coworkers reported that GFAP becomes detectable 24-72 $\mathrm{h}$ post-stroke, and steadily increases and peaks between 48 and $96 \mathrm{~h}$ [24].

In conclusion, this pilot study demonstrated that $\mathrm{NfH}^{\mathrm{SMI} 35}$ levels become detectable in the hyperacute phase of stroke. Differences in the early $\mathrm{NfH}^{\mathrm{SMI} 35}$ release pattern 
in ischaemic and haemorrhagic stroke could not be evaluated due to only two patients with the latter stroke entity. Further limitations include the small sample size, and lack of stratification for stroke localization (e.g. vascular territory, cortical vs. subcortical) and cause of ischaemic stroke. Indeed, biomarkers released by cortical pathology are likely to be found quicker and at higher concentration in the bloodstream due to diffusion through the cortical arachnoid villi [22]. Potential factors influencing $\mathrm{NfH}$ release include systemic blood pressure, haemodilution and brain temperature [26]. Glucose metabolism and glutamate toxicity are additional factors which could influence $\mathrm{NfH}$ release in the setting of cerebral ischemia. While no posthoc adjustments were possible due to the small sample size, these factors were closely monitored and variations kept at a minimum. The issues should be considered for the set-up of further studies aimed at refining the potential value and different time windows of serum $\mathrm{NfH}^{\text {SMI35 }}$ in future stroke management.

Acknowledgments JS is supported by a scientific fellowship and a Department to Department fellowship, both from the European Federation of Neurological Sciences (EFNS). This work was supported by a grant from The Stroke Association and was undertaken at UCLH/UCL who received a proportion of funding from the Department of Health's NIHR Biomedical Research Centres funding scheme. MMB's Chair in Stroke Medicine at University College London is supported by the Reta Lila Weston Trust for Medical Research. The authors would like to thank Prof. Geoffrey Keir for continuous support.

\section{References}

1. Macrez R, Ali C, Toutirais O et al (2011) Stroke and the immune system: from pathophysiology to new therapeutic strategies. Lancet Neurol 10:471-480

2. Brunkhorst R, Pfeilschifter W, Foerch C (2011) Astroglial proteins as diagnostic markers of acute intracerebral hemorrhagepathophysiological background and clinical findings. Transl Stroke Res 1:246-251

3. Foerch C, Montaner J, Furie KL et al (2009) Invited article: searching for oracles? Blood biomarkers in acute stroke. Neurology 73:393-399

4. Dvorak F, Haberer I, Sitzer M et al (2009) Characterisation of the diagnostic window of serum glial fibrillary acidic protein for the differentiation of intracerebral haemorrhage and ischaemic stroke. Cerebrovasc Dis 27:37-41

5. Petzold A, Michel P, Stock M et al (2008) Glial and axonal body fluid biomarkers are related to infarct volume, severity, and outcome. J Stroke Cerebrovasc Dis 17:196-203

6. Foerch C, Singer OC, Neumann-Haefelin T et al (2005) Evaluation of serum S100B as a surrogate marker for long-term outcome and infarct volume in acute middle cerebral artery infarction. Arch Neurol 62:1130-1134

7. Wunderlich MT, Wallesch CW, Goertler M (2004) Release of neurobiochemical markers of brain damage is related to the neurovascular status on admission and the site of arterial occlusion in acute ischemic stroke. J Neurol Sci 227:49-53
8. Dassan P, Keir G, Brown MM (2009) Criteria for a clinically informative serum biomarker in acute ischaemic stroke: a review of S100B. Cerebrovasc Dis 27:295-302

9. Petzold A (2005) Neurofilament phosphoforms: surrogate markers for axonal injury, degeneration and loss. J Neurol Sci 233: 183-198

10. Petzold A, Thompson EJ, Keir G et al (2009) Longitudinal oneyear study of levels and stoichiometry of neurofilament heavy and light chain concentrations in CSF in patients with multiple system atrophy. J Neurol Sci 279:76-79

11. Singh P, Yan J, Hull R et al (2011) Levels of phosphorylated axonal neurofilament subunit $\mathrm{H}(\mathrm{pNfH})$ are increased in acute ischemic stroke. J Neurol Sci 304:117-121

12. Heiss WD, Sobesky J, Hesselmann V (2004) Identifying thresholds for penumbra and irreversible tissue damage. Stroke 35: $2671-2674$

13. Lu M, Mitsias PD, Ewing JR et al (2005) Predicting final infarct size using acute and subacute multiparametric MRI measurements in patients with ischemic stroke. J Magn Reson Imaging 21:495-502

14. Jensen HA, Loukogeorgakis S, Yannopoulos F et al (2011) Remote ischemic preconditioning protects the brain against injury after hypothermic circulatory arrest. Circulation 123: 714-721

15. Sellner J, Petzold A, Sadikovic S et al (2009) The value of the serum neurofilament protein heavy chain as a biomarker for perioperative brain injury after carotid endarterectomy. Neurochem Res 34:1969-1974

16. Petzold A, Keir G, Green AJ et al (2004) An ELISA for glial fibrillary acidic protein. J Immunol Methods 287:169-177

17. Petzold A, Keir G, Lim D et al (2003) Cerebrospinal fluid (CSF) and serum S100B: release and wash-out pattern. Brain Res Bull 61:281-285

18. Lama S, Qiao M, Kirton A et al (2011) Imaging corticospinal degeneration in neonatal rats with unilateral cerebral infarction. Exp Neurol 228:192-199

19. Vexler ZS, Yenari MA (2009) Does inflammation after stroke affect the developing brain differently than adult brain? Dev Neurosci 31:378-393

20. Foerch C, Wunderlich MT, Dvorak F et al (2007) Elevated serum S100B levels indicate a higher risk of hemorrhagic transformation after thrombolytic therapy in acute stroke. Stroke 38:2491-2495

21. Missler U, Wiesmann M, Friedrich C et al (1997) S-100 protein and neuron-specific enolase concentrations in blood as indicators of infarction volume and prognosis in acute ischemic stroke. Stroke 28:1956-1960

22. Eikelenboom MJ, Uitdehaag BMJ, Petzold A (2011) Blood and CSF biomarker dynamics in multiple sclerosis: implications for data interpretation. Mult Scler Int Article ID 823176. doi: 10.1155/2011/823176

23. Petzold A, Mondria T, Kuhle J et al (2010) Evidence for acute neurotoxicity after chemotherapy. Ann Neurol 68:806-815

24. Herrmann M, Vos P, Wunderlich MT et al (2000) Release of glial tissue-specific proteins after acute stroke: a comparative analysis of serum concentrations of protein S-100B and glial fibrillary acidic protein. Stroke 31:2670-2677

25. Jauch EC, Lindsell C, Broderick J et al (2006) Association of serial biochemical markers with acute ischemic stroke: the national institute of neurological disorders and stroke recombinant tissue plasminogen activator stroke study. Stroke 37: $2508-2513$

26. Petzold A, Tisdall MM, Girbes AR et al (2011) In vivo monitoring of neuronal loss in traumatic brain injury: a microdialysis study. Brain 134:464-483 